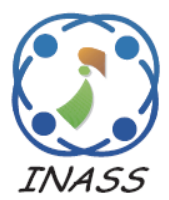

\title{
The Structural Modeling of Significant Factors for Sustainable Cloud Migration
}

\author{
Naim Ahmad ${ }^{1 *}$ \\ ${ }^{1}$ Department of Information Systems, King Khalid University, Saudi Arabia \\ * Corresponding author's Email: nagqadir@kku.edu.sa
}

\begin{abstract}
Cloud computing is a matured platform to host the information and communication technology (ICT) infrastructure and services. All the top leading companies have made investments in cloud infrastructure including US Department of Defence's contract of USD 10 Billion to Microsoft for cloud migration. Cloud computing helps in conserving the resources and improving the performance of ICT services, hence making the computing more sustainable. Migration to cloud needs careful planning, strong business case, credible migration strategy and robust migration frameworks. This research has reviewed the area of cloud computing migration to identify important factors. The author keywords of 299 research articles downloaded from the web of science database have been analyzed using co-word analysis and resulted in 10 most significant factors in the form of cloud migration tuple. This study applies the interpretive structural modeling (ISM) technique to study the interrelationship on these factors. Application of ISM followed by Matriced' Impacts Croise's Multiplication Appliquée a UN Classement (MICMAC) analysis has categorized these factors into independent and dependent sets. The resultant model demonstrates that organizations need to incorporate the dimensions of service orientation, virtualization, multi utilization, dynamic allocation and mobile support into their data, application and security stacks. Migration of these stacks with sound strategy, policy and frameworks will lead to post-migration performance improvements in a synergistic fashion. The adoption of cloud migration tuple with understanding of interrelationships will help organizations make strategies and plan to migrate to cloud in an efficient manner.
\end{abstract}

Keywords: Cloud computing migration, Cloud migration tuple, Interpretive structural modeling (ISM), Matrice d'impacts Croise's Multiplication Appliquée a UN Classement (MICMAC), Sustainable cloud adoption.

\section{Introduction}

Cloud computing "offers scalable or elastic computing on virtually complete range of computing devices, supporting all existing and archaic software technologies and tools, and served through disparate network hence making it platform independent, portable and ubiquitous" [1]. Public cloud service revenue is growing in the areas of cloud application services or Software as a Service (SaaS), cloud business process services (BPaaS), cloud system infrastructure services or Infrastructure as a Service (IaaS), cloud application infrastructure services or Platform as a Service (PaaS), cloud management and security services [2]. And, leading companies with their cloud services are Microsoft (SaaS, IaaS and PaaS), Amazon (IaaS and PaaS), IBM (SaaS, IaaS and
PaaS), Salesforce (SaaS, PaaS) and SAP (SaaS, IaaS and PaaS) [3].

Cloud computing is deployed through public, private, community and hybrid clouds, and offered in service delivery models such as IaaS, PaaS, SaaS etc. It utilizes virtualization to offer unified interface and services from the numerous hardware resources through virtual machine monitors (VMM) or hypervisors. The type-1 or bare metal hypervisors work directly on the hardware to manage virtual machines (VM) such as XEN, Microsoft Hyper-V, Oracle VM Server, VMware ESX and ESXi. And type-2 or hosted hypervisors that run on top of an operating system such as Redhat KVM and VMware Workstation [4]. Whereas, cloud services are offered through web services designed on web services description language (WSDL) and simple object access protocol (SOAP) and universal description, 
discovery, and integration (UDDI) industry standards [5]. And inside the cloud, they are managed and orchestrated through service-oriented architecture (SOA).

Similarly, containers virtualization technology is being used by large cloud providers such Google, IBM/Softlayer and Joyent [6]. Mostly containers utilize Linux kernel containment features, LXC. Containers share same host operating system (OS) therefore are lighter and hundreds of them can be run on single hardware machine. Whereas, on top of hypervisors, each VM can have its different OS. Containers provide the view of underlying OS to developers unlike VMs that have complete implementation of OS. Dockers help in the deploying Linux application inside containers. And Google's Kubernetes manages the cluster of docker containers.

Cloud computing also provides a new approach to application development and deployment known as cloud native. Cloud native approach among others ensures the stateless computing so that elasticity of cloud can be achieved in real-time and hence user traffic can be dynamically directed to any server regardless of their state of sessions. Cloud-native application (CNA) "is a distributed, elastic and horizontal scalable system composed of (micro) services which isolates state in a minimum of stateful components. The application and each self-contained deployment unit of that application is designed according to cloud-focused design patterns and operated on a self-service elastic platform" [7].

The global cloud infrastructure causes latency and jitters and fog computing helps overcome it. Fog computing is defined as "an architecture that uses edge devices to carry out a substantial amount of computation, storage, communication locally and routed over the internet backbone" [8]. Fog computing is a standard that facilitates the edge computing and is highly desirable in the mobile computing and Internet of things (IoT) applications to overcome the problems of latency and jitter, context awareness and voluminous data.

Cloud computing helps in achieving the objectives of sustainable computing. Sustainability is a very holistic performance measure and defined as "development that meets the needs of the present without compromising the ability of future generations to meet their needs" [9]. Cloud computing allows for maximum resource utilization through virtualization and multitenancy. Still corporations see many challenges to migrate to the cloud due to issues related to security, data management, governmental regulations, cloud computing competency, migration strategy and recovery of ICT assets in times of disaster or switching the cloud service provider.
The adoption of cloud have been studied through antecedent approaches [10-12]. Also there exist studies that present cloud adoption toolkits that help in making decisions for cloud migration [13]. In the organizational context behavioural studies attempt to illustrate the process of cloud migration [14]. Similarly, migration theory has also been adopted to study the process of cloud migration [15]. There also exists cloud simulation tools such SimGrid (https://simgrid.org) that help in experimenting with cloud environment and adopted in some studies [16, 17].

The objective of this paper is to identify interrelationships between significant factors of cloud migration identified in cloud migration tuple [18]. The cloud migration tuple was arrived through literature review and author keyword analysis, utilizing the concept of co-word analysis as reported in [18]. This research applied interpretive structure modeling (ISM) technique to develop interrelationships between these factors with the help of experts. ISM is a computer assisted learning technique and is being applied in many IS and sustainability research $[19,20]$. Also, MICMAC analysis has been carried out to get more understanding on the factors. Remaining of the paper is organized as research methodology, cloud migration tuple, article description, ISM and MICMAC analysis, discussions, and conclusion, limitation and future research.

\section{Research methodology}

The objective of this research is to develop the structural model of significant factors of cloud migration using state of the art literature and ISM. In order to collect the research articles in the area of cloud computing migration, Web of Science database was used. As this database contains the high-quality peer reviewed research papers. The query for the keyword "cloud computing migration framework" resulted in 299 research articles. Their details are given in the article description section.

Co-word analysis is a technique to analyze the significant words or keywords to study the conceptual structure of a research field [21]. In this research the authors' keywords of the 299 articles have been studied using concept of co-word analysis to identify the significant factors present in the context of cloud computing migration. Author keywords are extracted into the spreadsheet software and cluster of related terms are formed. Clusters having more than 10 items have been studied, defined and formed into a cloud migration tuple as illustrated in corresponding section.

ISM has been applied to develop model of interrelationships among elements of cloud migration 
tuple. ISM is a computer assisted learning technique that uses Boolean matrix algebra and graph theory [22-26]. Certain number of decision makers (DMs) or domain experts compare pair-wise variables for one of the four possible relationships (such as for variables $a$ and $b$ ) either $a$ influences $b, b$ influences $a, a$ and $b$ influence each other, or $a$ and $b$ don't influence each other. Based on the input of DMs further steps of ISM are carried out as explained in ISM application section. In the present study four DMs participated in the brainstorming session. They were all from academics having more than 10 years of experience, one associate processor, one $\mathrm{PhD}$ lecturer and two lecturers pursuing $\mathrm{PhD}$.

ISM is generally coupled with MICMAC analysis. MICMAC analysis helps in classifying the variables into four categories autonomous, independent, linkage and dependent [27]. The analysis helps in setting prioritization for handling dependent variables as they will affect the independent variables.

\section{Article description}

The articles were between the time period of 2009 to 2018 as shown in Table 1. Maximum articles were published in the year of 2015. Table 2 shows the publishing sources having frequency count 5 or more.

Table 1. Year-wise Publication Frequency

\begin{tabular}{lll}
\hline Publication years & Article count & Bar chart \\
\hline 2018 & 37 & \\
2017 & 46 & \\
2016 & 55 & \\
2015 & 60 & \\
2014 & 34 & \\
2013 & 29 & \\
2012 & 24 & \\
2011 & 12 & \\
2009 & 2 & \\
\hline
\end{tabular}

Table 2. High Frequency Publication Sources

\begin{tabular}{lc}
\hline \multicolumn{1}{c}{ Source of publication } & Count \\
\hline Lecture Notes in Computer Science & 9 \\
IEEE International Conf. on Cloud Computing & 7 \\
Future Generation Computer Systems & 6 \\
Journal of Network and Computer Applications & 6 \\
Communications in Computer and Information & 5 \\
Science & 5 \\
IEEE Access & 5 \\
International Conf. on Cloud Computing & 5 \\
Technology and Science & 5 \\
Journal of Supercomputing & \\
\hline
\end{tabular}

\section{Cloud Migration Tuple}

Cloud migration tuple was identified through coword analysis and clustering of author keywords present in 299 articles in the area of cloud migration [18]. There were total of 1263 keywords and 768 unique keywords. Clusters having 10 or more related terms were considered to derive most important dimensions. Ten such clusters were identified. The identified clusters of related terms are as follows virtualization, service orientation, data, multi utilization, dynamic allocation, mobile support, performance, security, application and migration [18] These terms are explained in the context of cloud computing in the following paragraphs.

Virtualization: this term is very integral of cloud computing as the whole technology works on virtualization of resources. Similarly, the virtual machine (VM) related concepts and processes have also appeared in this cluster such as VM consolidation, migration, live migration, placement, provisioning, storage migration, scheduling and security [28-32]. Moreover, virtual network [33,34] and virtual data center [35] also happen to fall under this cluster.

Service Orientation: it is also a unique characteristics of cloud computing as it is delivered as a service. Some important terms related are service orientation, availability, innovation, migration, portability and replication [36-38]. Service oriented architecture deserves a mention here as well. And service level agreements (SLA) are also one the most researched topic in this cluster and some related terms are SLA assurance and monitoring [37, 39].

Data: data is primary resource of information technology. Many important terms are mentioned with data such as locality, distribution, portability, migration, security, mining and deduplication [32, 40-42]. Data centers (DC) are the basic infrastructural component of cloud computing that contain data. Therefore DC networking and management also deserves mention here as well [43].

Multi Utilization: multi term has many-faceted implication in cloud computing. Most common of all is multi-tenant environment. Multi-Agent system (system of multiple interacting intelligent system) [44] has also become significant in the context of cloud computing. Similarly some underlying concepts that help in multi-utilization are replication and redundancy such as multicast [43], multi-cloud [45] and multi-gateway [46] system.

Dynamic Allocation: dynamic term is associated with real-time changes and requires application of advanced algorithms. For instance, to ensure elasticity, dynamic resource allocation capability of cloud native applications is important. Similarly, dynamism is desired for load balancing [47], consolidation [30], priority, resource migration and 
scheduling [48]. This construct is also important for dynamic migration and consolidation of VMs, and dynamic structures [49].

Mobile Support: mobile support is provided by all the cloud service providers given the pervasiveness of smart phones and devices [50]. Within this concept mobile [50] and edge computing [51] become essential to solve the problems of jitter and latency. Additionally, the term mobile is used in conjunction with network [52], platform [53] and services [54].

Performance: this is the most important criterion for the success of cloud computing migration. This term has both, pre-migration importance such as performance matrix [55], modelling [56], prediction [57] and testing [58]. Similarly, in the post migration stage performance attributes [47], analysis [59], evaluation and management [60] is of great significance to assess migration success.

Security: security and privacy has been the most debated and stumbling block for migration to the cloud. Security is important in all the aspects such as migration, services and data. Security needs careful planning. Security requirements [61], metrics and measurements [62]should be well defined. This term is also used in combination with compliance [62] and transparency [63]. Security also needs to be maintained during live migration of VMs [64, 65].

Application: in the traditional sense software were said to be applications rather than services, and older applications are today termed as legacy applications. Application migration and offloading [50, 66] are important concepts in the cloud computing context. And for application or code offloading frameworks such as AppSpecCloudlet [67] for specific cloudlet selection are included in this cluster. Similarly, postmigration concepts of application adaptation and optimization are of great importance [68]. Some other important terms are application replication and application-aware allocation.

Migration: migration refers to moving organizations ICT infrastructure to the cloud infrastructure. Migration may follow on of the approach namely rehost, re-platform, re-factor or rebuild [69, 70]. Similarly, migration policy, methodology and framework need to be developed well in advance before migration process execution. Migration patterns [71] also assist in managing the migration.

There are some variables that are very innate of cloud computing environment such as virtualization, multi utilization and dynamic allocation. Whereas, cloud is service based architecture and encourages applications to migrate onto the service oriented or microservices architectures to achieve maximum performance [72]. Integration of mobile support is easier in cloud infrastructure than in the traditional IT infrastructure by virtue of mobile cloud computing. Whereas, Data and security issues exists both in traditional and cloud computing infrastructure. Data and applications are supposed to be migrated to the cloud infrastructure in anticipation of performance enhancements. Therefore, set of variables logically represent all the important aspects of cloud computing migration.

\section{ISM and MICMAC analysis}

Interpretive structural modeling is a computer assisted learning tool. The first step is to identify the factors in the domain of study and carried out through literature review and co-word analysis on the author keywords as explained in the previous section. In the second step, a brainstorming session is conducted with the help of four domain experts for the pair-wise comparison of variables to establish contextual relationships. One of the four symbols V, A, X and O were placed in the structural self-interaction matrix (SSIM) for two variables $a$ and $b$ indicating one of the four relations, $a$ influences $b, b$ influences $a$, $a$ and $b$ influence each other, or $\mathrm{a}$ and $\mathrm{b}$ don't influence each other respectively Table 3 . For example, as per experts' verdict virtualization will influence migration, application, security, performance, mobile support and data; will mutually influence dynamic allocation and multi utilization; and will be influenced by service orientation. Accordingly, V, X, and A symbols have been entered in the first row of Table 3. Likewise, for all constructs, symbols have been entered as per experts' opinions. SSIM servers the basic input for other steps of ISM such as reachability matrix, final reachability matrix incorporating transitivity, level partitioning, conical matrix, diagraph and final model. For developing reachability matrix, $\mathrm{V}, \mathrm{A}, \mathrm{X}$ and $\mathrm{O}$ symbols are replaced with binary digits 0 or 1 based upon following rules.

Let $m_{i j}$ be the element of SSIM and $r_{i j}$ be the element of reachability matrix where $i$ and $j$ represent row and column index.

$$
\begin{aligned}
& \text { if } \mathrm{m}_{\mathrm{ij}}=\mathrm{V} \text { then } \mathrm{r}_{\mathrm{ij}}=1 \& \mathrm{r}_{\mathrm{ji}}=0 \\
& \text { if } \mathrm{m}_{\mathrm{ij}}=\mathrm{A} \text { then } \mathrm{r}_{\mathrm{ij}}=0 \& \mathrm{r}_{\mathrm{ji}}=1 \\
& \text { if } \mathrm{m}_{\mathrm{ij}}=\mathrm{X} \text { then } \mathrm{r}_{\mathrm{ij}}=1 \& \mathrm{r}_{\mathrm{ji}}=1 \\
& \text { if } \mathrm{m}_{\mathrm{ij}}=\mathrm{O} \text { then } \mathrm{r}_{\mathrm{ij}}=0 \& \mathrm{r}_{\mathrm{ji}}=0
\end{aligned}
$$

Hence obtained reachability matrix is further checked for transitive reachability meaning if $\mathrm{x}$ leads to $\mathrm{y}$ and $\mathrm{y}$ leads to $\mathrm{z}$ then $\mathrm{x}$ will also lead to $\mathrm{z}$. All the transitive relations are represented by $1^{*}$ and the resultant matrix is called final reachability matrix Table 4. This table also shows the driving and 
dependence powers by adding non-zero rows and columns respectively.

Following step estimates the ranks or levels of the variable through level partitioning. For each variable reachability and antecedent, and their intersection sets are calculated. Variables corresponding to non-zero row elements including variable gives the reachability set and similarly column for antecedent set. For any variable if intersection set matches with the reachability set then that variable is given top level or Level I and removed for the next iteration from all the sets. Iteration continues until all variables are assigned the levels. In this case, within four iteration all variables were assigned level from I to IV, Table 5.

In the next step, the conical matrix is formed after sorting the final reachability matrix based on the levels of variables row and column wise. Conical matrix helps in making diagraph that is drawn by representing nodes corresponding to variables and directed edges based on non-zero cells in conical matrix. Finally, the diagraph is converted into final model by removing unnecessary transitive links and replacing node ids with corresponding variable details Fig. 2.

Matriced' Impacts Croise's Multiplication Appliquée a UN Classement (MICMAC) analysis helps in categorizing variables into autonomous, dependent, linkage and independent classes. Based on the dependence and driving power (Table 4), all variables are mapped onto two by two matrix Fig. 2 .

Table 3. Matrix Indicating Structural Self-Interaction

\begin{tabular}{|c|c|c|c|c|c|c|c|c|c|c|}
\hline No & Elements & 10 & 9 & 8 & 7 & 6 & 5 & 4 & 3 & 2 \\
\hline 1 & Virtualization & $\mathrm{V}$ & $\mathrm{V}$ & V & $\mathrm{V}$ & $\mathrm{V}$ & $\mathrm{X}$ & $\mathrm{X}$ & $\mathrm{V}$ & $\bar{A}$ \\
\hline 2 & $\begin{array}{l}\text { Service } \\
\text { Orientation }\end{array}$ & V & $\mathrm{O}$ & V & V & $\mathrm{V}$ & $\mathrm{V}$ & V & V & \\
\hline 3 & Data & V & V & A & V & A & $\mathrm{O}$ & A & & \\
\hline 4 & $\begin{array}{l}\text { Multi } \\
\text { Utilization }\end{array}$ & V & $\mathrm{O}$ & V & V & $\mathrm{V}$ & A & & & \\
\hline 5 & $\begin{array}{l}\text { Dynamic } \\
\text { Allocation }\end{array}$ & V & $\mathrm{O}$ & $\mathrm{O}$ & V & V & & & & \\
\hline 6 & Mobile Support & $\mathrm{V}$ & V & V & V & & & & & \\
\hline 7 & Performance & A & $\mathrm{V}$ & A & & & & & & \\
\hline 8 & Security & A & A & & & & & & & \\
\hline 9 & Application & $\mathrm{A}$ & & & & & & & & \\
\hline 10 & Migration & & & & & & & & & \\
\hline
\end{tabular}

Table 4. Final reachability matrix

\begin{tabular}{clccccccccccc}
\hline S. No. & \multicolumn{1}{c}{ Elements } & $\mathbf{1}$ & $\mathbf{2}$ & $\mathbf{3}$ & $\mathbf{4}$ & $\mathbf{5}$ & $\mathbf{6}$ & $\mathbf{7}$ & $\mathbf{8}$ & $\mathbf{9}$ & $\mathbf{1 0}$ & Driving Power \\
\hline 1 & Virtualization & 1 & 0 & 1 & 1 & 1 & 1 & 1 & 1 & 1 & 1 & 9 \\
2 & Service Orientation & 1 & 1 & 1 & 1 & 1 & 1 & 1 & 1 & $1^{*}$ & 1 & 10 \\
3 & Data & 0 & 0 & 1 & 0 & 0 & 0 & 1 & $1^{*}$ & 1 & 1 & 5 \\
4 & Multi Utilization & 1 & 0 & 1 & 1 & $1^{*}$ & 1 & 1 & 1 & $1^{*}$ & 1 & 9 \\
5 & Dynamic Allocation & 1 & 0 & $1^{*}$ & 1 & 1 & 1 & 1 & $1^{*}$ & $1^{*}$ & 1 & 9 \\
6 & Mobile Support & 0 & 0 & 1 & 0 & 0 & 1 & 1 & 1 & 1 & 1 & 6 \\
7 & Performance & 0 & 0 & $1^{*}$ & 0 & 0 & 0 & 1 & $1^{*}$ & 1 & $1^{*}$ & 5 \\
8 & Security & 0 & 0 & 1 & 0 & 0 & 0 & 1 & 1 & $1^{*}$ & $1^{*}$ & 5 \\
9 & Application & 0 & 0 & $1^{*}$ & 0 & 0 & 0 & $1^{*}$ & 1 & 1 & $1^{*}$ & 5 \\
10 & Migration & 0 & 0 & $1^{*}$ & 0 & 0 & 0 & 1 & 1 & 1 & 1 & 5 \\
& Dependence & 4 & 1 & 10 & 4 & 4 & 5 & 10 & 10 & 10 & 10 & \\
\hline
\end{tabular}

Table 5. Variables' levels through iteration I - IV

\begin{tabular}{ccccc}
\hline $\begin{array}{c}\text { Elements } \\
(\mathbf{M i})\end{array}$ & $\begin{array}{c}\text { Reachability } \\
\text { R(Mi) set }\end{array}$ & Antecedent set A(Ni) & $\begin{array}{c}\text { Intersection set, } \\
\text { R(Mi) } \cap \mathbf{A}(\mathbf{N i})\end{array}$ & Level \\
\hline 1 & $1,4,5$, & $1,2,4,5$, & $1,4,5$, & III \\
2 & 2, & 2, & 2, & IV \\
3 & $3,7,8,9,10$, & $1,2,3,4,5,6,7,8,9,10$, & $3,7,8,9,10$, & I \\
4 & $1,4,5$, & $1,2,4,5$, & $1,4,5$, & III \\
5 & $1,4,5$, & $1,2,4,5$, & $1,4,5$, & III \\
6 & 6, & $1,2,4,5,6$, & 6, & II \\
7 & $3,7,8,9,10$, & $1,2,3,4,5,6,7,8,9,10$, & $3,7,8,9,10$, & I \\
8 & $3,7,8,9,10$, & $1,2,3,4,5,6,7,8,9,10$, & $3,7,8,9,10$, & I \\
9 & $3,7,8,9,10$, & $1,2,3,4,5,6,7,8,9,10$, & $3,7,8,9,10$, & I \\
10 & $3,7,8,9,10$, & $1,2,3,4,5,6,7,8,9,10$, & $3,7,8,9,10$, & I \\
\hline
\end{tabular}




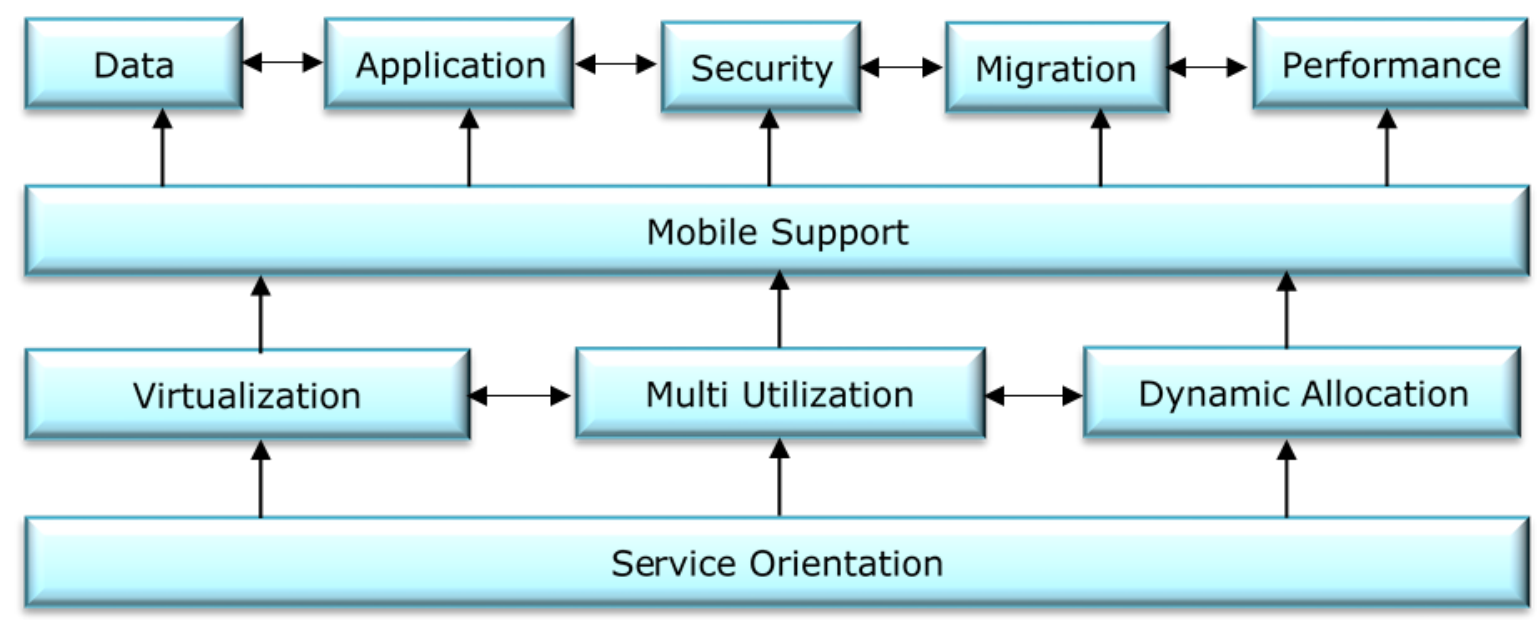

Figure. 1 ISM based model for cloud computing migration

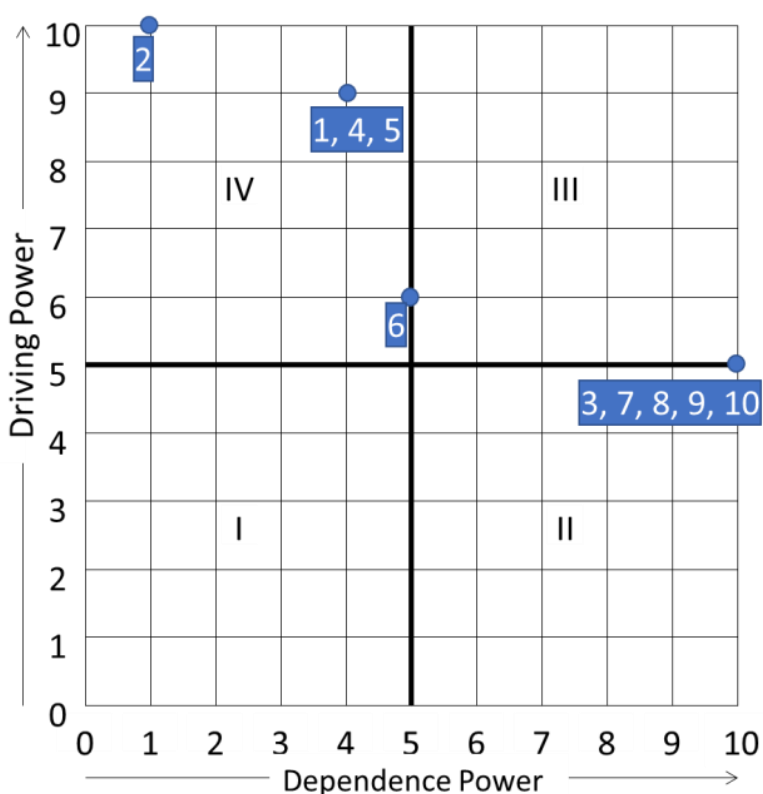

I-Autonomous Variables II-Dependent Variables III-Linkage Variables IV-Independent Variables

Figure. 2 Driving power and dependence diagram

\section{Discussion}

Cloud migration is on the top of agenda for most of the corporations. Cloud computing brings in sustainability in the IT services and operations, due to optimal utilization of resources. Organizations needs to be aware of the technological evolution behind cloud computing so as to make informed decision to adopt it. Cloud computing trends section illustrates upon the hypervisor, container, cloud-native and fog computing.

Traditionally cloud offered compute and storage services through virtualization without any care for bandwidth. To solve this issue, the concept of virtual network and data centres is gaining momentum. Recent research is focusing more onto developing algorithms and mechanisms to migrate VMs into different data centres for energy and bandwidth optimizations. Another emerging research area is application offloading, essential in the context of mobile cloud computing. Similarly, frameworks such as organizational sustainability modelling are useful tools for organizations to assess their investments in the cloud.

There exist numerous frameworks and tools to assist in cloud migration. This research provides ten most significant dimensions that need careful consideration for successful cloud computing adoption. These dimensions are service orientation, virtualization, multi utilization, dynamic allocation, mobile support, data, application, security, migration and performance. The list is in order of independent to dependent variables as per the outcome of ISM and MICMAC analysis.

ISM model and MICMAC analysis gives the interaction among identified factors in the context of cloud computing migration. Service orientation is the core of cloud computing in design, delivery and development. Therefore, organizations can't ignore migration of their IT artefacts into service model to utilize maximum benefit in the journey to cloud. For instance, legacy application migrated onto IaaS mode will sit on top of cloud rather being imbibed into the cloud infrastructure. Secondly the innate nature of the cloud is virtualization, multi-utilization and dynamic allocation of resources. These three dimensions influence each other in a synergistic fashion. And these primitive features of cloud also greatly influence cost, energy and security measures.

Mobile support has been achieved at a very mature level through mobile cloud computing. Organizations must have clear strategies to harness mobile cloud computing to extend IT services to the mobile devices. Post-migration performance will be greatly affected 
by incorporating mobile cloud computing. MICMAC analysis puts all these five factors as independent variables for the successful cloud migration.

Rest of the five factors, data, application, security, migration and performance are classified as dependent factors in the same level and influencing each other. Data, application and security stacks are migrated onto the cloud through different migration policies, strategies and frameworks. Such as lift and shift migration strategy (infrastructure as a service) will have implications on performance outcomes. Similarly, performance will impact the migration of data and application to the cloud. Security requirements will also dictate data and application, migration and performance. Therefore, these five dimensions needs to be managed in coordinated style.

\section{Conclusion, limitations and future research}

Cloud computing has become a norm of the day and all the leading organization commercial or government of different size and domain are adopting it, recent being the US Defence. Cloud computing technology is maturing day by day with hypervisors, containers, cloud native applications, flog computing, edge computing and cloudlets to become a robust platform to host complete range of information technology services. Cloud computing in the current state of the art is moving towards virtual network and data centres to solve bandwidth issues. Similarly, application offloading algorithms and frameworks have become recent research topics to support mobile cloud computing.

In this study, with the help of expert opinion and application of ISM and MICMAC analysis the constructs of cloud migration tuple are interrelated and grouped into independent and dependent categories. The independent variables are service orientation, virtualization, multi utilization, dynamic allocation and mobile support. Whereas, the dependent variables are data, application, security, migration and performance.

The results of this study illustrate that organizations need to incorporate the dimensions of service orientation, virtualization, multi utilization, dynamic allocation and mobile support into their data, application and security stacks. Migration of these stacks with sound strategy, policy and frameworks will lead to post-migration performance improvements in a synergistic fashion. Though a comprehensive, Web of science database was considered, in future more databases may be considered. In order to identify most significant factors only bigger clusters have been explained whereas, smaller cluster may shed lights to more niche areas. The model arrived may also be tested using some cloud simulation tools such as SimGrid.

\section{Conflicts of Interest}

The authors declare no conflict of interest.

\section{Acknowledgments}

The author wishes to thank King Khalid University, KSA and all the decision-makers for sparing their time to provide expert opinions.

\section{References}

[1] N. Ahmad, "Cloud computing: Technology, security issues and solutions", In: Proc. of 2017 2nd International Conf. on Anti-Cyber Crimes (ICACC), Abha, Saudi Arabia, pp. 30-35, 2017.

[2] Gartner, "Gartner Forecasts Worldwide Public Cloud Revenue to Grow 17.3 Percent in 2019", https://www.gartner.com/en/newsroom/pressreleases/2018-09-12-gartner-forecastsworldwide-public-cloud-revenue-to-grow-17percent-in-2019.

[3] Forbes, "The Top 5 Cloud-Computing Vendors: \#1 Microsoft, \#2 Amazon, \#3 IBM, \#4 Salesforce, \#5 SAP", https://www.forbes.com/sites/bobevans1/2017/1 1/07/the-top-5-cloud-computing-vendors-1microsoft-2-amazon-3-ibm-4-salesforce-5sap/\#45e113526f2e.

[4] S. Campbell and M. Jeronim, Applied Virtualization Technology, Intel Press, Hillsboro, USA, 2006.

[5] D.S. Linthicum, Cloud computing and SOA convergence in your enterprise: a step-by-step guide, Addison-Wesley Professional, Indianapolis, USA, 2009.

[6] D. Bernstein, "Containers and cloud: From lxc to docker to kubernetes", IEEE Cloud Computing, Vol. 1, No. 3, pp. 81-84, 2014.

[7] N. Kratzke and P.-C. Quint, "Understanding cloud-native applications after 10 years of cloud computing-a systematic mapping study", Journal of Systems and Software, Vol. 126, pp. 1-16, 2017.

[8] WikiPedia, "Fog Computing", https://en.wikipedia.org/wiki/Fog_computing.

[9] R. H. Cassen, Our Common Future, Report of the World Commission on Environment and Development, International Affairs, 1987.

[10] S.-K. Yoo and B.-Y. Kim, "A Decision-Making Model for Adopting a Cloud Computing 
System”, Sustainability, Vol. 10, No. 8, p. 2952, 2018.

[11] R. El-Gazzar, E. Hustad, and D.H. Olsen, "Understanding cloud computing adoption issues: A Delphi study approach", Journal of Systems and Software, Vol. 118, pp. 64-84, 2016.

[12] R. Rai, G. Sahoo, and S. Mehfuz, "Exploring the factors influencing the cloud computing adoption: a systematic study on cloud migration", SpringerPlus, Vol. 4, No. 1 p. 197, 2015.

[13] A. Khajeh-Hosseini, D. Greenwood, J.W. Smith, and I. Sommerville, "The cloud adoption toolkit: supporting cloud adoption decisions in the enterprise", Software: Practice and Experience, Vol. 42, No. 4, pp. 447-465, 2012.

[14] A. Alkhalil, R. Sahandi, and D. John, "An exploration of the determinants for decision to migrate existing resources to cloud computing using an integrated TOE-DOI model", Journal of Cloud Computing: Advances, Systems and Applications, Vol. 6, p. 2017.

[15] A. Bhattacherjee and S.C. Park, "Why end-users move to the cloud: a migration-theoretic analysis", European Journal of Information Systems, Vol. 23, No. 3, pp. 357-372, 2014.

[16] A. Kraemer, C. Maziero, O. Richard, and D. Trystram, "Reducing the number of response time service level objective violations by a cloud-HPC convergence scheduler", Concurrency and Computation: Practice and Experience, Vol. 30, No. 12, p. e4352, 2018.

[17] T. Hirofuchi, A. Lebre, and L. Pouilloux, "SimGrid VM: Virtual Machine Support for a Simulation Framework of Distributed Systems", IEEE Transactions on Cloud Computing, Vol. 6, No. 1, pp. 221-234, 2018.

[18] N. Ahmad, S. Qamar, N. Khan, et al., "Cloud Computing Trends and Cloud Migration Tuple", In: Proc. of Innovations in Electronics and Communication Engineering: H.S. Saini, R.K. Singh, M. Tariq Beg, J.S. Sahambi, Eds., Springer: Berlin, Germany, pp. 737-745, 2020.

[19] N. Ahmad, N. Quadri, M. Qureshi, and M. Alam, "Relationship Modeling of Critical Success Factors for Enhancing Sustainability and Performance in E-Learning", Sustainability, Vol. 10, No. 12, p. 4776, 2018.

[20] S. Khan, A. Haleem, M. Khan, M. Abidi, and A. Al-Ahmari, "Implementing traceability systems in specific supply chain management (SCM) through critical success factors (CSFs)", Sustainability, Vol. 10, No. 1, p. 204, 2018.

[21] M. Callon, J.-P. Courtial, W.A. Turner, and S. Bauin, "From translations to problematic networks: An introduction to co-word analysis",
Information (International Social Science Council), Vol. 22, No. 2, pp. 191-235, 1983.

[22] J.N. Warfield, An Assault on Complexity, Battelle Office of Corporate Communications, Columbus, USA, 1973.

[23] J.N. Warfield, "Developing subsystem matrices in structural modeling", IEEE Transactions on Systems, Man, and Cybernetics, Vol. 4, No. 1, pp. 74-80, 1974.

[24] J.N. Warfield, "Developing interconnection matrices in structural modeling", IEEE Transactions on Systems, Man, and Cybernetics, Vol. 4, No. 1, pp. 81-87, 1974.

[25] J.N. Warfield, Societal systems: Planning, Policy and Complexity, John Wiley \& Sons, New York, NY, USA, 1976.

[26] R. Attri, N. Dev, and V. Sharma, "Interpretive structural modelling (ISM) approach: an overview", Research Journal of Management Sciences, Vol. 2319, p. 1171, 2013.

[27] M. Godet, "Introduction to la prospective: seven key ideas and one scenario method", Futures, Vol. 18, No. 2, pp. 134-157, 1986.

[28] Z. Rehman, O.K. Hussain, and F.K. Hussain, "User-side cloud service management: State-ofthe-art and future directions", Journal of Network and Computer Applications, Vol. 55, pp. 108-122, 2015.

[29] G. Molto, M. Caballer, and C. de Alfonso, "Automatic memory-based vertical elasticity and oversubscription on cloud platforms", Future Generation Computer Systems, Vol. 56, pp. 1-10, 2016.

[30] K. Ye, Z. Wu, C. Wang, et al., "Profiling-Based Workload Consolidation and Migration in Virtualized Data Centers", IEEE Transactions on Parallel and Distributed Systems, Vol. 26, No. 3, pp. 878-890, 2015.

[31] S.K. Bose, S. Brock, R. Skeoch, N. Shaikh, and S. Rao, "Optimizing Live Migration of Virtual Machines Across Wide Area Networks Using Integrated Replication and Scheduling”, In: Proc. of 2011 IEEE International Systems Conf., Montreal, Canada, pp. 97-102, 2011.

[32] S.B. Rathod and V.K. Reddy, "NDynamic Framework for Secure VM Migration over Cloud Computing", Journal of Information Processing Systems, Vol. 13, No. 3, pp. 476-490, 2017.

[33] C. Liang and F.R. Yu, "Wireless network virtualization: A survey, some research issues and challenges", IEEE Communications Surveys \& Tutorials, Vol. 17, No. 1, pp. 358-380, 2015.

[34] A. Aissioui, A. Ksentini, A. (Mourad) Gueroui, and T. Taleb, "Toward Elastic Distributed 
SDN/NFV Controller for 5G Mobile Cloud Management Systems", IEEE Access, Vol. 3, pp. 2055-2064, 2015.

[35] M.F. Zhani, Q. Zhang, G. Simon, and R. Boutaba, "VDC Planner: Dynamic Migration-Aware Virtual Data Center Embedding for Clouds", In: Proc. of 2013 IFIP/IEEE International Symposium on Integrated Network Management (IM 2013), Ghent, Belgium, pp. 18-25, 2013.

[36] V. Chang, R.J. Walters, and G. Wills, "The development that leads to the Cloud Computing Business Framework", International Journal of Information Management, Vol. 33, No. 3, pp. 524-538, 2013.

[37] J. Pavlik, V. Sobeslav, and A. Komarek, "Measurement of Cloud Computing Services Availability", In: International Conf. on Nature of Computation and Communication: P. Vinh, E. Vassev, M. Hinchey Eds., Springer, Cham, pp. 191-201, 2015.

[38] M. Yingchi, W. Jiulong, Z. Lili, and J. Qing, "Optimization Service Migration Scheme for Load Balance in Cloud Computing", In: Proc. of International Conf. on Computer Science and Service System (CSSS 2014), Bangkok, Thailand, pp. 634-637, 2014.

[39] A.A.Z.A. Ibrahim, D. Kliazovich, and P. Bouvry, "On Service Level Agreement Assurance in Cloud Computing Data Centers", In: Proc. of 2016 IEEE 9th International Conf. on Cloud Computing (CLOUD), San Francisco, USA pp. 921-926, 2016.

[40] J. Li, B. Stephenson, H.R. Motahari-Nezhad, and S. Singhal, "GEODAC: A Data Assurance Policy Specification and Enforcement Framework for Outsourced Services", IEEE Transactions on Services Computing, Vol. 4, No 4, pp. 340-354, 2011.

[41] M. Sookhak, H. Talebian, E. Ahmed, A. Gani, and M.K. Khan, "A review on remote data auditing in single cloud server: Taxonomy and open issues", Journal of Network and Computer Applications, Vol. 43, pp. 121-141, 2014.

[42] F. Rashid, A. Miri, and I. Woungang, "Secure Enterprise Data Deduplication in the Cloud", In: Proc. of 2013 IEEE Sixth International Conf. on Cloud Computing, Santa Clara, USA, pp. 367374, 2013.

[43] G.M. Saridis, S. Peng, Y. Yan, et al., "Lightness: A function-virtualizable software defined data center network with all-optical circuit/packet switching", Journal of Lightwave Technology, Vol. 34, No. 7, pp. 1618-1627, 2016.

[44] S. Laghari and M.A. Niazi, "Modeling the Internet of Things, Self-Organizing and Other
Complex Adaptive Communication Networks: A Cognitive Agent-Based Computing Approach", PLoS One, Vol. 11, No. 1, p. 2016.

[45] J. Hadley, Y. Elkhatib, G. Blair, and U. Roedig, "MultiBox: Lightweight Containers for VendorIndependent Multi-cloud Deployments", In: Proc. of Workshop on Embracing Global Computing in Emerging Economies: R. Horne Eds., Springer, Cham, pp. 79-90, 2015.

[46] H. Feng and W. Wu, "Framework and User Migration Strategy of Cloud-Based Video Conference Multi-Gateway System", In: Proc. of 2012 19th International Conf. on High Performance Computing, Pune India, pp. 1-8, 2012.

[47] B. Addis, D. Ardagna, B. Panicucci, M.S. Squillante, and L. Zhang, "A Hierarchical Approach for the Resource Management of Very Large Cloud Platforms", IEEE Transactions on Dependable and Secure Computing, Vol. 10, No. 5, pp. 253-272, 2013.

[48] C.-W. Tsai and J.J.P.C. Rodrigues, "Metaheuristic Scheduling for Cloud: A Survey", IEEE Systems Journal, Vol. 8, No. 1, pp. 279291, 2014.

[49] B. Di Martino and A. Esposito, "Automatic Dynamic Data Structures Recognition to Support the Migration of Applications to the Cloud", International Journal of Grid and High Performance Computing, Vol. 7, No. 3, p. 2015.

[50] M. Shiraz, A. Gani, R.H. Khokhar, and R. Buyya, "A review on distributed application processing frameworks in smart mobile devices for mobile cloud computing", IEEE Communications Surveys \& Tutorials, Vol. 15, No. 3, pp. 1294 1313, 2013.

[51] O. Osanaiye, S. Chen, Z. Yan, R. Lu, K.-K.R. Choo, and M. Dlodlo, "From Cloud to Fog Computing: A Review and a Conceptual Live VM Migration Framework", IEEE Access, Vol. 5, pp. 8284-8300, 2017.

[52] S.-H. Hung, C.-S. Shih, J.-P. Shieh, C.-P. Lee, and Y.-H. Huang, "Executing mobile applications on the cloud: Framework and issues", Computers \& Mathematics with Applications, Vol. 63, No. 2, pp. 573-587, 2012.

[53] L. Martinez, C. Pereira, and L. Favre, "Migrating $\mathrm{C} / \mathrm{C}++$ Software to Mobile Platforms in the ADM Context", International Journal of Interactive Multimedia and Artificial Intelligence, Vol. 4, No. 3, pp. 34-44, 2017.

[54] T.H. Noor, S. Zeadally, A. Alfazi, and Q.Z. Sheng, "Mobile cloud computing: Challenges and future research directions", Journal of 
Network and Computer Applications, Vol. 115, pp. 70-85, 2018.

[55] K. Nagamani, C.G.V.N. Prasad, and K.S. Chatrapati, "A Novel Framework for Optimal Component Based Data Center Architecture", In: Proc. of 2016 International Conf. on Information Communication and Embedded Systems (ICICES), Chennai, India, pp. 1-8, 2016

[56] W. Zhang, K.T. Lam, and C.-L. Wang, "Adaptive Live VM Migration over a WAN: Modeling and Implementation", In: Proc. of 2014 IEEE 7th International Conf. on Cloud Computing, Anchorage. USA, pp. 368-375, 2014.

[57] M. Atif and P. Strazdins, "Adaptive parallel application resource remapping through the live migration of virtual machines", Future Generation Computer Systems, Vol. 37, pp. 148-161, 2014.

[58] L. Zhang, Y. Chen, F. Tang, and X. Ao, "Design and Implementation of Cloud-based Performance Testing System for Web Services", In: Proc. of 2011 6th International ICST Conf. on Communications and Networking in China (CHINACOM), Harbin, China, pp. 875-880 2011.

[59] E. Ahmed, A. Akhunzada, M. Whaiduzzaman, A. Gani, S.H. Ab Hamid, and R. Buyya, "Networkcentric performance analysis of runtime application migration in mobile cloud computing", Simulation Modelling Practice and Theory. Vol. 50, pp. 42-56, 2015.

[60] O.A. Oral and B. Tekinerdogan, "OneService Generic Cache Aggregator Framework for Service Depended Cloud Applications", In: Proc. of 2017 IEEE 10th International Conf. on Cloud Computing (CLOUD), Honolulu, USA, pp. 640-647, 2017.

[61] C. Kalloniatis, H. Mouratidis, and S. Islam, "Evaluating cloud deployment scenarios based on security and privacy requirements", Requirements Engineering, Vol. 18, No. 4, pp. 299-319, 2013.

[62] J. Luna, H. Ghani, D. Gemianus, and N. Suni, "A security metrics framework for the cloud", In: Proc. of the International Conf. on Security and Cryptography, Seville, Spain. pp. 245-250 (2011).

[63] U.M. Ismail, S. Islam, M. Ouedraogo, and E. Weippl, "A Framework for Security Transparency in Cloud Computing", Future Internet, Vol. 8, No. 1, p. 5, 2016.

[64] T. Huang, Y. Zhu, Y. Wu, S. Bressan, and G. Dobbie, "Anomaly detection and identification scheme for VM live migration in cloud infrastructure", Future Generation Computer Systems, Vol. 56, pp. 736-745, 2016.

[65] M. Kashkoush, M. Azab, M. Eltoweissy, and G. Attiya, "Towards Online Smart Disguise: Realtime Diversification Evading Co-Residency Based Cloud Attacks", In: Proc. of 2017 IEEE 3rd International Conf. on Collaboration and Internet Computing (CIC), San Jose, USA, pp. 235-242 (2017).

[66] M. Shiraz and A. Gani, "A lightweight active service migration framework for computational offloading in mobile cloud computing", The Journal of Supercomputing, Vol. 68, No. 2, pp. 978-995, 2014.

[67] D.G. Roy, D. De, A. Mukherjee, and R. Buyya, "Application-aware cloudlet selection for computation offloading in multi-cloudlet environment", The Journal of Supercomputing, Vol. 73, No. 4, pp. 1672-1690, 2017.

[68] V. Andrikopoulos, T. Binz, F. Leymann, and S. Strauch, "How to adapt applications for the Cloud environment", Computing, Vol. 95, No. 6, pp. 493-535, 2013.

[69] Gartner, "Gartner Identifies Five Ways to Migrate Applications to the Cloud", https://www.gartner.com/newsroom/id/1684114.

[70] N. Ahmad, Q.N. Naveed, and N. Hoda, "Strategy and procedures for Migration to the Cloud Computing", In: Proc. of 2018 IEEE 5th International Conf. on Engineering Technologies and Applied Sciences (ICETAS), Bangkok, Thailand, pp. 1-5, 2018.

[71] P. Jamshidi, C. Pahl, and N.C. Mendonça, "Pattern-based multi-cloud architecture migration", Software: Practice and Experience, Vol. 47, No. 9, pp. 1159-1184, 2017.

[72] P. Jamshidi, A. Ahmad, and C. Pahl, "Cloud migration research: a systematic review", IEEE Transactions on Cloud Computing, Vol. 1, No. 2, pp. 142-157, 2013. 